\title{
Estados de ánimo pre y post competitivos en atletas chilenos universitarios de alto rendimiento
}

\author{
Pre- and post-competitive mood states in chilean university \\ high-performance athletes
}

\author{
*Francisco Gallardo Fuentes, *Rodrigo Ramírez Campillo, **Roberto Sáez Lafourcade, \\ **Jorge Gallardo Fuentes, **Cristian Aguilar Ruiz, ***Bastian Carter Thuillier, \\ *Angélica Castillo Cerda, *Cristian Álvarez
}

Gallardo, F., Ramírez, R., Sáez, R., Gallardo, J., Aguilar, C., Carter, B., Castillo, A., Álvarez, C. (2019). Estados de ánimo pre y post competitivos en atletas chilenos universitarios de alto rendimiento. Revista Ciencias de la Actividad Física $U C M, \mathrm{~N}^{\circ} 20$ (1), enero-junio, 1-10. DOI: http://doi.org/10.29035/rcaf.20.1.7

\section{RESUMEN}

El presente estudio describe el comportamiento de los estados de ánimo en deportistas de alto rendimiento, pertenecientes a un equipo de atletismo universitario en etapa de final nacional. Para este efecto se encuestaron a través del instrumento Profile of Mood States (POMS) en su versión adaptada, a veinticinco deportistas, previo (pretest) e inmediatamente después (postest) de haber competido en la final nacional de las Ligas Deportivas Universitarias de Chile. El test consta de 51 adjetivos que a su vez se agrupan en cinco grandes factores: tensión, depresión, cólera, vigor y fatiga. En el pretest los factores que presentan un mayor puntaje en la escala de 0-4 son: vigor (2.59), tensión (1.57), seguidos de fatiga (.99), cólera (.43) y depresión (.34). En el postest: vigor (1.66) y fatiga (1.39), seguidos de cólera (.66), tensión (.64) y depresión (.33). Se encontraron diferencias significativas (en base al valor $\mathrm{p} \leq .05$ ) en el pretest vs. postest para tensión y vigor, con valores más altos en ambos casos para el pretest. En conclusión, los factores de tensión y vigor son más elevados previos a la competición, disminuyendo posterior a la competición. Estos resultados podrían ayudar a entrenadores y profesionales en la preparación de los deportistas frente a competiciones importantes.

\section{PALABRAS CLAVE}

Estados de ánimo, atletas, alto rendimiento, atletas universitarios.

\begin{abstract}
The present study describes the behavior of mood states in high performance athletes belonging to a University team in the national championship. For this purpose, twenty-five athletes were surveyed using the "Profile of Mood States" (POMS) instrument, in their adapted version, before (pretest) and immediately after (post-test) competing in the national final of the University Sports Leagues of Chile. The test consisted of 51 adjectives, grouped into five big factors: tension, depression, anger, vigor and fatigue. In the pretest, the factors that presented a higher score on the scale of 0 -4 were: vigor (2.59), tension (1.57), followed by fatigue (.99), anger (.43) and depression (.34). In the posttest: vigor (1.66) and fatigue (1.39), followed by anger (.66), tension (.64) and depression (.33). Significant differences were found (on the basis of the value $\mathrm{p} \leq .05$ ) in the pretest vs. post-test for tension and vigor, with higher values in both cases than in the pretest. In conclusion, the tension and vigor factors are higher before to the competition, decreasing after the competition. These results could help coaches and professionals in the athletes' preparation for important competitions.
\end{abstract}

\section{Key words}

Mood States, Athletes, High-Performance, University Athletes.

* Departamento de Ciencias de la Actividad Física. Universidad de los Lagos, Osorno, Chile.

** Investigador independiente. Osorno, Chile.

*** Universidad Católica de Temuco. Temuco, Chile. 


\section{INTRODUCCIÓN}

La relevancia que ha alcanzado la actividad física y el deporte en diferentes sociedades ha llevado a la ciencia a interactuar con mayor frecuencia y seriedad desde múltiples áreas, esto ya que desde diferentes disciplinas es posible contar con reportes que asocian la práctica de actividad física y deportes con efectos positivos; podemos encontrar mejoras en los estilos de vida (Castillo, Balaguer y GarcíaMerita, 2007), mejoras documentadas en algunos indicadores de salud fisiológica como los niveles de presión arterial (Iza-Stoll, 2006); así también se ha encontrado una asociación entre la baja práctica de actividad física con la presencia de obesidad, y enfermedades como la diabetes mellitus (Varo, Martínez y Martínez-González, 2003). Fruto de la proliferación de estudios en el ámbito de la actividad física y el deporte, se puede ver un incremento en las revistas de divulgación científica del área; en España, ya en el año 2003, mediante un estudio se logró identificar 26 revistas científico técnicas del área de las ciencias de la actividad física y el deporte (Devis-Devis, Antolín, Villamon, Moreno y Valenciano, 2003).

Dentro del área actividad física y deporte existen varias sub especialidades que estudian fenómenos asociados, entre ellas la psicología, la cual plantea que la práctica regular de actividad física puede prevenir enfermedades mentales, ya que las personas son menos susceptibles a los factores desencadenantes de estas (Márquez, 1995). Ahora, más específicamente en el área de la actividad física y el deporte, la psicología deportiva estudia aspectos como el control del estrés y la autoconfianza, con la obtención de éxito y continuidad a largo plazo en el deporte de competición (Gimeno, Buceta y Pérez-Llanta, 2012), así como estudios que basados en las interacciones que las variables psicológicas tienen en el rendimiento deportivo, con propuestas como la implementación de programas de entrenamiento psicológico (Nieto y Olmedilla, 2007).

En cuanto al deporte competitivo, las interacciones que ayuden a la mejora del rendimiento son fundamentales, entre ellas la interacción deportista-psicología deportiva
(Mora, Zarco y Blanca, 2001; Reyes, Julia y Gómez, 2012). Dentro de las variables psicológicas que influyen en el rendimiento deportivo más estudiadas se encuentran los estados de ánimo. Estos generalmente son menos intensos, más persistentes y menos dirigidos que las emociones, y están presentes en los momentos claves de la competencia (Totterdell, 1999). Por tanto, resulta relevante descubrir qué factores serán los que influyen en los deportistas para alcanzar sus metas, de manera que se busca dar con aquellos que son significativos y demuestran generar estados de ánimo positivos para la superación de metas y aumento del rendimiento.

El instrumento más utilizado para determinar los estados de ánimo en situaciones deportivas es el perfil de los estados de ánimo, más conocido por sus siglas en inglés, Profile of Mood States (POMS) (McNair, Lorr, \& Droppleman, 1971). Este instrumento se viene utilizando hace muchos años como medición valida, ya en el año 2002 Andrade-Fernández, Arce-Fernández y Seaone-Pesqueira indican que: "desde su introducción en el ámbito del deporte, en 1975, ha habido al menos 315 publicaciones relacionadas con el POMS" (2002, p. 709).

Se pueden encontrar múltiples investigaciones que utilizan el POMS como medida válida para determinar los estados de ánimo, es así que experiencias que utilizaran el POMS como el estudio de Arruza, Arribas, Otaegi, González, Irazusta y Ruiz (2011), en una muestra de 30 deportistas considerados como promesas deportivas en 10 disciplinas diferentes, entregan reportes de correlaciones entre el nivel de los deportistas y el nivel de las dimensiones generadoras de estrés, es decir, los deportistas de mayor nivel competitivo presentan valores más bajos en las dimensiones generadoras de estrés. Así también otro estudio en el que participaran 3 deportistas medallistas europeos y olímpicos, a los que se les realizo un seguimiento en 98 competiciones previo a los juegos olímpicos de Atlanta 96, utilizando el POMS entre 25 a 35 min previo a la competición, se encontraron con que los niveles de fatiga percibida pueden ser un buen predictor del rendimiento deportivo (Arruza, Balagué y Arrieta, 1998). 
En base a lo antes descrito, se puede afirmar que los estados de ánimo cumplen un rol importante en el rendimiento deportivo, incluso influyendo en el mismo (Molina, Sandín y Chorot, 2014) y encontrándose sobre todo una relación negativa entre la ansiedad y el rendimiento (Craft, Magyar, Becker y Feltz, 2003; Pinto y Vázquez, 2013; Scanlan, Babes y Scanlan, 2005). A pesar de todo, no se han encontrado investigaciones que entreguen reportes en torno a las variaciones ocurridas previo y posterior a la instancia competitiva, por lo que resulta importante evidenciar si hay fluctuaciones en la percepción de los estados de ánimo de los deportistas, sobre todo con una cercana inmediatez, tanto previo, como posterior a la competencia. Por tanto, el objetivo principal de este estudio fue describir los estados de ánimo pre y post competencia y determinar el efecto de la competencia sobre estos.

\section{MATERIALES Y MÉTODOS}

El diseño metodológico utilizado en el presente estudio corresponde a un estudio de casos intrínseco, ya que, como dice Stake (2010) este tipo de diseño metodológico entiende que al estudiar una realidad desde su propia manifestación, esta se convierte y representa un caso en sí misma. La prueba POMS fue aplicada a un grupo $(n=25)$ de atletas universitarios de alto rendimiento, participantes de la final de las Ligas Deportivas de Educación Superior en Chile. La edad de los competidores se sitúa entre los 18 y 30 años, de los cuales el $40 \%$ son mujeres y $60 \%$ hombres, competidores en las pruebas del atletismo tales como: velocidad, medio fondo, fondo, marcha, saltos y lanzamientos. Es importante mencionar que este estudio contó con la aprobación individualizada de los participantes, además de estar sujetos al comité de ética del Departamento de Ciencias de la Actividad Física de la Universidad de los Lagos de Osorno que declara pertinente su aplicación. Los sujetos fueron entrevistados durante los cinco minutos previos a realizarse la competencia e inmediatamente después ( $\sim 10$ minutos) de haber finalizado la prueba. En las pruebas que contaban con fases clasificatorias y final, se les aplicó el cuestionario en ambas instancias, pero para efectos de análisis de datos del presente estudio se utilizó solo el instrumento aplicado en el nivel más avanzado de la competición. Si bien el cuestionario fue auto-suministrado, el investigador principal resolvió cualquier inquietud por parte de los deportistas durante la aplicación del instrumento.

Resaltar que el instrumento se validó a través de la aplicación de un Alpha de Cronbach, tanto en la prueba previa como en la prueba posterior a las competiciones.

En el presente estudio se utilizó la adaptación de (Balaguer, Fuentes, Meliá, García-Mérita y Pons, 1994). En dicho estudio se analizó el POMS y su estructura factorial, después de dicho análisis se redujeron los factores, con esto, el factor de confusión y el factor de amistad quedaron fuera y la prueba POMS quedó compuesta de cinco factores.

La prueba quedó configurada, a partir del análisis factorial, por cinco factores y 51 adjetivos; el instrumento se presenta como cuestionario contando con una escala tipo Likert de cinco grados, donde: $0=$ nada; $1=$ un poco; $2=$ moderadamente; $3=$ bastante; $4=$ muchísimo, en respuesta a los 51 adjetivos que se presentan (ver Tabla 1). La prueba POMS nos permite generar una relación dinámica entre los factores psicológicos que influyen en la práctica de deportes de alto rendimiento, mediante la aplicación de este test previo y posterior a la competencia. La definición de cada uno de los factores que considera el POMS elaborado a partir de Belaguer et al. (1993) es:

“El factor D(Depresión) parece representar un estado de ánimo deprimido, acompañado de un sentimiento de inadecuación personal. Los adjetivos que lo componen son: infeliz, dolido, triste, abatido, desesperanzado, torpe, desanimado, solo, desdichado, deprimido, desesperado, desamparado, inútil, aterrorizado y culpable.

El factor A (Cólera) representa un estado de ánimo de cólera y antipatía hacia los demás. Los adjetivos son: enfadado, enojado, irritable, resentido, molesto, rencoroso, amargado, luchador, rebelde, decepcionado, furioso y de mal genio. 
El factor T (Tensión) se define por adjetivos que describen incrementos en la tensión músculo-esquelética: tenso, agitado, a punto de estallar, descontrolado, relajado, intranquilo, inquieto, nervioso y ansioso.

El factor $V$ (Vigor) representa un estado de vigor, ebullición y energía elevada. Está formado por los siguientes adjetivos: animado, activo, enérgico, alegre, alerta, lleno de energía, despreocupado y vigoroso.

El factor $F$ (Fatiga) representa un estado de ánimo de laxitud, inercia, y bajo nivel de energía. Formados por los adjetivos: agotado, apático, fatigado, exhausto, espeso, sin fuerzas y cansado" (Belaguer et al., 1993, p, 5-6).

A su vez cada factor estaba conformado por una serie de adjetivos (Tabla 1).

\section{Tabla 1}

Factores y adjetivos de la prueba POMS.

\begin{tabular}{cl}
\hline Factores & Adjetivos \\
\hline $\begin{array}{c}\text { Depresión } \\
\text { (21 adjetivos) }\end{array}$ & $\begin{array}{l}\text { Desdichado, infeliz, amargado, des- } \\
\text { amparado, deprimido, triste, solo, } \\
\text { desanimado, desesperanzado, desespe- } \\
\text { rado, decepcionado, abatido, aturdido, } \\
\text { desorientado, inútil, dolido por actos } \\
\text { pasados, inseguro, confundido, aterro- } \\
\text { rizado, culpable y resentido. }\end{array}$ \\
\hline Cólera & $\begin{array}{l}\text { Enojado, enfadado, furioso, de mal } \\
\text { genio, molesto, irritable, rencoroso, a } \\
\text { punto de estallar* y rebelde. }\end{array}$ \\
\hline Tensión & $\begin{array}{l}\text { Nervioso, inquieto, intranquilo, tenso, } \\
\text { agitado, relajado, ansioso y a punto de } \\
\text { estallar* }\end{array}$ \\
\hline Vigor & $\begin{array}{l}\text { Enérgico, lleno de energía, vigoroso, } \\
\text { activo, luchador, alegre, animado, efi- } \\
\text { ciente y alerta. }\end{array}$ \\
\hline
\end{tabular}

Fatiga Fatigado, cansado, agotado, exhausto y (5 adjetivos) sin fuerza.

* Adjetivo que se repite en más de un factor.

\section{Análisis Estadísticos}

Los datos fueron recopilados y manejados a través del software estadístico Statistical Package for the Social Sciences (SPSS, versión 15.0). Los resultados del "cuestionario POMS" fueron sometidos a análisis de consistencia in- terna mediante Alpha de Cronbach; también se analizaron mediante estadística descriptiva, con la obtención de la media aritmética $(M)$ y la desviación típica $(d t$.). Los datos se sometieron a test al de normalidad de Shapiro Wilk; posteriormente las medias fueron sometidas a la prueba de $U$ de Mann-Whitney $(U)$ en búsqueda de diferencia de medias entre el pre y post-test de la "Prueba POMS", tomando como valor de significancia $p \leq .05$.

\section{RESULTADOS}

Con la finalidad de validar internamente la prueba aplicada en los 25 atletas de alto rendimiento, para el caso de este estudio en particular, se aplicó un Alpha de Cronbach, se observó una fiabilidad elevada ( $\geq .70$ en el factor tensión $\mathrm{y} \geq .80$ en los otros 4 factores), por lo que en términos rigurosos se ha permitido validar la consistencia interna de la aplicación y los resultados obtenidos a partir de la prueba POMS (Tabla 2).

\section{Tabla 2}

Factores y adjetivos de la prueba POMS.

\begin{tabular}{ccc}
\hline Factores & $\begin{array}{c}\text { Alpha Cronbach } \\
\text { Pre }\end{array}$ & $\begin{array}{c}\text { Alpha Cronbach } \\
\text { Post }\end{array}$ \\
\hline Depresión & .84 & .95 \\
\hline Cólera & .82 & .93 \\
\hline Tensión & .67 & .69 \\
\hline Vigor & .81 & .89 \\
\hline Fatiga & .93 & .89 \\
\hline
\end{tabular}

Se realizó un análisis de escala, para determinar fiabilidad en cada una de las dimensiones en el Pre y Post cuestionario, a través del índice Alpha de Conbrach.

Los resultados son observados a partir de las puntuaciones obtenidas en la prueba POMS aplicada a los 25 deportistas incluidos. Se puede apreciar que los factores que presentaron mayor heterogeneidad representada en los valores más elevados de sus desviaciones típicas fueron, tanto en el pretest (.47/.53), como en el postest (.51/.46), los de tensión y vigor (Tabla 3). 


\section{Tabla 3}

Medias y dt. Previo y posterior a la competición en "la prueba POMS" (valor medio, por factor) (*indica diferencias significativas).

\begin{tabular}{ccccccc}
\hline & \multicolumn{2}{c}{ Pretest } & \multicolumn{2}{c}{ Postest } & \multicolumn{2}{c}{ Pre vs. post } \\
\cline { 2 - 7 } & $M$ & $d t$. & $M$ & $d t$. & $U$ & Sig. \\
\hline Depresión & .34 & .20 & .50 & .33 & 154 & .937 \\
\hline Cólera & .43 & .18 & .66 & .36 & 24 & .144 \\
\hline Tensión & 1.57 & .47 & .64 & .51 & 6 & ${ }^{\star} .005$ \\
\hline Vigor & 2.59 & .53 & 1.66 & .46 & 8 & ${ }^{*} .004$ \\
\hline Fatiga & .99 & .23 & 1.39 & .35 & 4 & .758 \\
\hline
\end{tabular}

En la Tabla 3, se puede apreciar una tendencia en el pretest hacia una alta valoración a los factores tensión (1.57) y vigor (2.59), ambos con puntuaciones en el rango entre 1 y 3 (un poco/ bastante), y bajas puntuaciones, también en el pretest, para los factores depresión (.34), cólera (.43) y fatiga (.99), todos con puntuaciones entre 0 y 1 (nada/ un poco).

Situación similar se puede apreciar en el postest con una alta valoración para el factor vigor (1.66) que se ubica entre 1 y 2 (un poco/ moderadamente), por otro lado nos encontramos con una puntuación más baja comparada a la del pretest para el factor tensión (.64), y los factores de depresión (.50) y cólera (.66) se mantuvieron con puntuaciones bajas entre $0 \mathrm{y}$ 1 (nada/ un poco). Finalmente el factor fatiga (1.39) recibió puntuaciones superiores al pretest, esta vez entre 1 y 2 (un poco/ moderadamente).

Con respecto al análisis de diferencia de medias del pretest vs. postest se puede ver que las puntuaciones para los diferentes factores se comportó con valoraciones más elevadas en el pretest para tensión (1.57 vs. .64) y vigor (2.59 vs. 1.66), y más elevadas en el postest vs. pretest para depresión (.34 vs. .50), cólera (.43 vs. .66) y fatiga (.99 vs. 1.39). Sin embargo, solo encontramos diferencias de medias significativas en 2 casos: tensión y vigor, ambos factores que presentaran estas diferencias de media significativa, presentaron valores más elevados en el pretest.

En la Figura 1, se aprecia el comportamiento de las curvas que componen el perfil Iceberg.

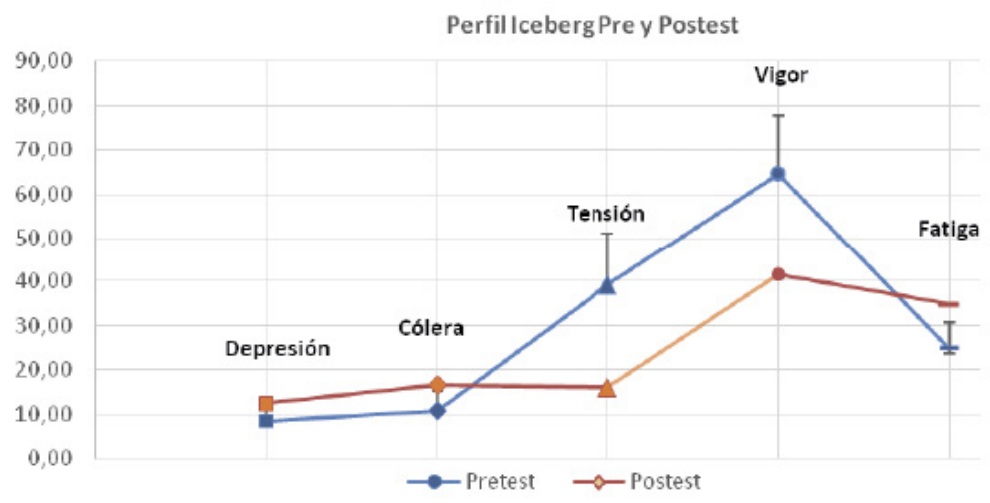

Figura 1. Perfil Iceberg del pretest y postest.

Como se aprecia en la Figura 1, el perfil iceberg que se desprende del análisis de la prueba POMS se manifestó especialmente en el pretest como un estado acorde a lo que se esperaría para deportistas, considerando lo que manifiesta Andrade, Arce y Seoane-Pesqueira (2000), ya que el factor de vigor se sitúa por encima de los demás factores. 


\section{DISCUSIÓN}

La fiabilidad de la prueba POMS se corroboró mediante la prueba estadística Alpha de Cronbach, encontrándose cuatro dimensiones con valores superiores a los .80, tanto en el pretest como en el postest, esto se sitúa estrechamente ligado al concepto de validez que va en respuesta a la pregunta ¿está usted midiendo lo que usted cree que está midiendo? Y como bien lo define (Kerlinger, 1992) "Validez, en términos generales, se refiere al grado en que un instrumento realmente mide la variable que pretende medir", lo que deja ver una alta consistencia interna en los datos obtenidos, al igual se han podido constatar resultados similares en otras experiencias (Palacios, González, Arruza, Arribas y Irasusta, 2015), lo que motivó a elegir el instrumento. Además de sus antecedentes históricos, considerando que ya en el año 1980, Morgan decía: "de todos los tests psicológicos que mis colegas y yo hemos probado, el POMS ha demostrado ser el mejor predictor del éxito deportivo" (p. 97).

$\mathrm{Al}$ analizar las diferencias de medias el pretest vs. postest, estas presentaron una marcada contraposición entre el factor vigor (2.59 vs. 1.66) y el factor fatiga (.99 vs. 1.39). De manera que vigor se presentó más elevado en el pretest y más bajo en el postest, al contrario de lo que ocurre con fatiga, en esta se puede apreciar que en otras experiencias se suelen encontrar estos mismos resultados, donde el factor vigor tiende a disminuir después de que el deportista se enfrenta a estímulos psicológicos y fisiológicos, y, por otra parte, la fatiga tiende a ser más elevada una vez se ha sometido el deportista a este tipo de estímulos (Filaire, Maso, Degoutte, Jouanel y Lac, 2001; Umeda et al., 2008).

Con respecto a las diferencias de media significativas entre pretest y postest, estas se presentaron solo en dos factores (vigor y tensión), ambos arrojaron valores con medias significativamente más bajas en el postest (ósea posterior a la competición), aunque en ambas situaciones estos factores fueron los más elevados; esto se puede deber al hecho de que la muestra correspondía a deportistas de alto rendimiento. Autores han constatado que personas físicamente activas obtienen puntuaciones por sobre la media para parámetros positivos (como vigor) (Morgan, Costill, Flynn, Raglin y O'Connor, 1988; Raglin, 1990), al contrastar los resultados con población similar a la del estudio (deportistas competitivos). Este postulado es reforzado ya que se puede ver que previo a la competición, los deportistas suelen presentar valores elevados en estos factores (vigor y tensión) (Hernández, Torres-Luque y Olmedilla, 2009), así, también hay quienes comentan que ganar o perder representa exigencias objetivas, y que estas son relativamente inestables e incontrolables, lo que además podría generar estados anímicos negativos en deportistas (Roberts, 1992), pero también hay experiencias como la de Cecchini, González, Carmona y Contreras (2004), quienes manifestaran que deportistas con una predominante orientación a la tarea no son generalmente susceptibles a la ansiedad competitiva, pues poseen estándares internos de ejecución y el resultado por el que se esfuerzan es subjetivo y relativamente controlable, sin embargo, Singh y Gaurav (2011) encontraron en su estudio una diferencia significativa en la ansiedad de jugadores y jugadoras de voleibol previo y posterior a instancias competitivas. Estas experiencias sitúan como muy relevante la aplicación de este tipo de estudios en deportistas de alta competencia en busca de conocer las manifestaciones de los estados de ánimo.

Aunque el estudio de los estados de ánimo suele utilizarse en contextos deportivos competitivos, también se pueden encontrar experiencias que relevan la importancia de los estados de ánimo en población que realiza actividad física no competitiva con fines de salud, donde se pueden apreciar mejores estados de ánimo y sensaciones de bienestar subjetivo (Martinsen, 2005), también experiencias como la que analizó el comportamiento de los estados de ánimo durante un programa de actividad física acuática para mujeres embarazadas, encontrándose con una baja paulatina del factor vigor y tensión a medida que pasaban las sesiones (Torres-Luque, Torres-Luque, Sánchez y Gutiérrez, 2010). Con toda la bibliografía analizada, consideramos que los estados de ánimo son además de suma importancia en 
instancias de iniciación deportiva, en donde se debe cuidar el no acumular experiencias que incidan negativamente en una excesiva ansiedad o un estado de ánimo negativo, ya que estos podrían acabar apartando al joven de la práctica del deporte.

Finalmente se pudo visualizar de manera gráfica las fluctuaciones del estado de ánimo en el prestest y el postest, estas fluctuaciones graficas se presentan a través del perfil iceberg, el que arrojó un valor más elevado para el factor vigor en ambas ocasiones, con coincidencias a lo que se entiende como un perfil de ánimo de un deportista (Andrade et al., 2000). Podemos también apreciar que posterior a la competencia existe un comportamiento que es distinto en los factores tensión y fatiga, vale decir, mientras la tensión baja, posterior a la competencia, la fatiga se eleva. Estas fluctuaciones en el estado de ánimo ha adquirido tal importancia que, incluso, se ha encontrado relación entre el sobre entrenamiento y algunas modificaciones en el perfil de los estados de ánimo (Andrade et al., 2000; Morgan, Costill, Flyenn, Raglin y O’Connor, 1988).

\section{CONCLUSIONES}

A modo de conclusión se puede apreciar que los momentos de la competencia están caracterizados por diferencias en los estados de ánimo; en el test previo a la competencia se observó que algunos determinados estados de ánimo se manifiestan por sobre otros (dadaS las diferencias entre factores), estados que, a su vez, al momento de terminar la competencia manifiestan diferencias, que en algunos casos presentan significancia estadística. Esto nos permite concluir que los estados de ánimo están presentes en la superación de una competencia, así como también en la no superación. Lo anterior reafirma que, independiente del resultado obtenido, la instancia competitiva genera variaciones en los estados de ánimo.

Las limitaciones del estudio responden al hecho que en las consideraciones ético-metodológicas del mismo, se planteó aplicar el cuestionario de forma anónima, lo que no permitió cruzar los resultados deportivos con los esta- dos de ánimo, por lo que, para futuros estudios, se recomienda considerar la posibilidad de generar un cruce entre POMS y rendimiento de los deportistas, con la finalidad de comprobar la incidencia de los estados de ánimo en los resultados competitivos en escenarios universitarios de alto rendimiento, o mejorar la información respecto de los estados de ánimo midiendo la orientación hacia el ego y la tarea con instrumentos como el "Taskand Ego Orientation in Sport Questionnaire" (TEOSQ).

\section{Aplicaciones Prácticas}

El presente estudio es aplicable en sujetos que persiguen rendimiento deportivo, y se puede instalar como una forma de controlar los estados de ánimo de deportistas enfrentados a la competencia. Esta potencialidad es de gran relevancia considerando que contar con instrumentos que permitan conocer las variables psicológicas como el estado de ánimo, son de gran ayuda para comprender cómo afronta el deportista las instancias competitivas, y permitiría, además, tomar las acciones idóneas casos de aparición de estados de ánimo negativos que interfieran con el rendimiento. 


\section{REFERENCIAS BIBLIOGRÁFICAS}

Andrade, E., Arce, C., y Seoane-Pesqueira, G. (2000). Aportaciones del POMS a la medida del estado de ánimo de los deportistas. Revista de Psicología del Deporte, 9(1-2), 07-20.

Andrade-Fernández, E., Arce-Fernández, C., y Seaone-Pesqueira, G. (2002). Adaptación al español del cuestionario «Perfil de los Estados de Ánimo» en una muestra de deportistas. Psicothema, 14(4). 708-713.

Arruza, J., Arribas, S., Otaegi, O., González, O., Irazusta, S., y Ruiz, L. (2011). Percepción de competencia, estado de ánimo y tolerancia al estrés en jóvenes deportistas de alto rendimiento. Anales de psicología, 27(2), 536-546.

Arruza, J., Balagué, G., y Arrieta, M. (1998). Rendimiento deportivo e influencia del estado de ánimo, de la dificultad estimada, y de la autoeficacia en la alta competición. Revista de psicología del deporte, 7(2), 193-204.

Balaguer, I., Fuentes, I., Meliá, J., García-Merita, M., y Pérez Recio, G. (1993). El perfil de los estados de ánimo (POMS): Baremo para estudiantes valencianos y su aplicación en el contexto deportivo. Revista de Psicología del deporte, 2(2), 39-52.

Balaguer, I., Fuentes, I., Meliá, J., García-Mérita, M., y Pons, D. (1994). Adaptación del Perfil de Estados de Ánimo (POMS) a una muestra de estudiantes valencianos. Trabajo presentado al IV Congreso de Evaluación Psicológica. Santiago de Compostela.

Castillo, I., Balaguer, I., y García-Merita, M. (2007). Efecto de la práctica de actividad física y de la participación deportiva sobre el estilo de vida saludable en la adolescencia en función del género. Revista de psicología del deporte, 16(2), 201-2010.
Cecchini, J., González, C., Carmona, Á. y Contreras, O. (2004). Relaciones entre clima motivacional, la orientación de meta, la motivación intrínseca, la autoconfianza, la ansiedad y el estado de ánimo en jóvenes deportistas. Psicothema, 16(1), 104-109.

Craft, L., Magyar, T., Becker, B. y Feltz, D. (2003). The relationship between the Competitive State Anxiety Inventory-2 and sport performance: A metaanalysis. Journal of Sport and Exercise Psychology, 25(1), 44-65. doi:10.1016/ j.psychsport.2008.08.001

Devis-Devis, J., Antolin, L., Villamon, M., Moreno, A., y Valenciano, J. (2003). Las revistas científico-técnicas españolas de las ciencias de la actividad física y el deporte: Inventario y análisis de la calidad de contenido y difusión. Revista española de documentación científica, 26(2), 177-190.

Filaire, E., Maso, F., Degoutte, F., Jouanel, P., y Lac, G. (2001). Food restriction, performance, psychological state and lipid values in judo athletes. International journal of sports medicine, 22(06), 454459.

Gimeno, F., Buceta, J., y Pérez-Llanta, M. (2012). El cuestionario «características psicológicas relacionadas con el rendimiento deportivo» (CPRD): Características psicométricas. Análise Psicológica, 19(1), 93-113.

Hernández, R., Torres-Luque, G., y Olmedilla, A. (2009) Relations among training volume, body weight and profile of mood states for elite judoka during a competitive period. Perceptual and Motor Skills, 109(3), 870-880.

Iza-Stoll, A. (2006). Tratamiento de la hipertensión arterial primaria. Acta Médica Peruana, 23(2), 93-99.

Kerlinger, F. (1992). Investigación del Comportamiento. México: McGraw-Hill. 
Márquez, S. (1995). Beneficios psicológicos de la actividad física. Revista de psicología general y aplicada, 48(1), 185-206.

Martinsen, E. (2005). Exercise and Depression. International Journal of Sport and Exercise Psychology, 3(4), 469-483. doi: $\underline{10.1080 / 1612197 X .2005 .10807319}$

McNair, D,. Lorr, M., y Droppleman, L. (1971). Profile of mood state manual. San Diego (CA): Educational and Industrial Testing Service.

Molina, J., Sandín, B., y Chorot, P. (2014). Sensibilidad a la ansiedad y presión psicológica: Efectos sobre el rendimiento deportivo en adolescentes. Cuadernos de psicología del deporte, 14(1), 45-54.

Mora, J., Zarco, J., y Blanca, M. (2001). Atención-concentración como entrenamiento para la mejora del rendimiento deportivo en jugadores profesionales de fútbol. Revista de psicología del deporte, 10(1), 49-65.

Morgan, W. (1980). Test of champions the iceberg profile. Psychology Today, 14(2), 92-99.

Morgan, W., Costill, D., Flynn, M., Raglin, J., y O'Connor, P. (1988). Mood disturbance following increased training in swimmers. Medicine and science in sports and exercise, 20(4), 408-414.

Nieto, G., y Olmedilla, A. (2007). Planificación del entrenamiento psicológico en atletas de élite: un caso en marcha atlética. Revista de Psicología del Deporte, 10(1), 127-142. Recuperado de: https:// www.rpd-online.com/article/viewFile/154/154.

Palacios, M., González, O., Arruza, J., Arribas, S. y Irasusta, S. (2015). Evaluación de la invarianza factorial entre las versiones lápiz/papel y online del profile of mood state (poms) en una muestra de deportistas. Revista mexicana de psicología, 32(1), 48-56.
Pinto, M., y Vázquez, N. (2013). Ansiedad estado competitiva y estrategias de afrontamiento: su relación con el rendimiento en una muestra argentina de jugadores amateurs de golf. Revista de Psicología del Deporte, 22(1), 47-52

Raglin, J. (1990) Exercise and mental health. Beneficial and detrimental effects. Sport Medicine, 9(6), 323-329.

Reyes, M., Julia, M., y Gómez, L. (2012). Programa de Entrenamiento en Habilidades Psicolgógicas en jugadoras de voleibol de alto rendimiento. Cuadernos de Psicología del Deporte, 12(1), 9-16.

Roberts, G. (1992). Motivation in sport and exercise: Conceptual constraints and convergence. Champaign, IL: Human Kinetics.

Scanlan, T., Babkes, M., y Scanlan, L. (2005). Participation in sport: A developmental glimpse at emotion. Organized activities as contexts of development: Extracurricular activities, after-school and community programs, 275-309.

Singh, A., y Gaurav, V. (2011). A study of pre-competitive and post-competitive anxiety level of inter-collegiate volleyball players. International Journal of Sports Science and Engineering, 5(04), 237-241.

Stake, R. (2010). Qualitative research: Studying how things work. New York, USA: Guilford Press.

Torres-Luque, G., Torres-Luque, L., Sánchez, M., y Gutiérrez, C. (2010). Empleo del POMS durante un programa de actividad física en el medio acuático para mujeres embarazadas. Cuadernos de psicología del deporte, 10(1).37-45

Totterdell, P. (1999). Mood scores: Mood and performance in professional cricketers. British Journal of Psychology, 90(3), 317-332. 
Umeda, T., Yamai, K., Takahashi, I., Kojima, A., Yamamoto, Y., Tanabe, M., Totsuka, M., Naksji, S., Sugawara, N., y Matsuzaka, M. (2008). The effects of a two hour judo training session on the neutrophil immune functions in university judoists. Luminescence, 23(1), 49-53.

Varo, J., Martínez, J., y Martínez-González, M. (2003). Beneficios de la actividad física y riesgos del sedentarismo. Medicina clínica, 121(17), 665-672.

\section{Dirección para correspondencia}

Francisco Javier Gallardo Fuentes

Académico Departamento de Ciencias de la

Actividad Física

Universidad de los Lagos

Osorno, Chile.

Contacto:

fgallardo@ulagos.cl

Recibido: 12-07-2018

Aceptado: 07-05-2019 